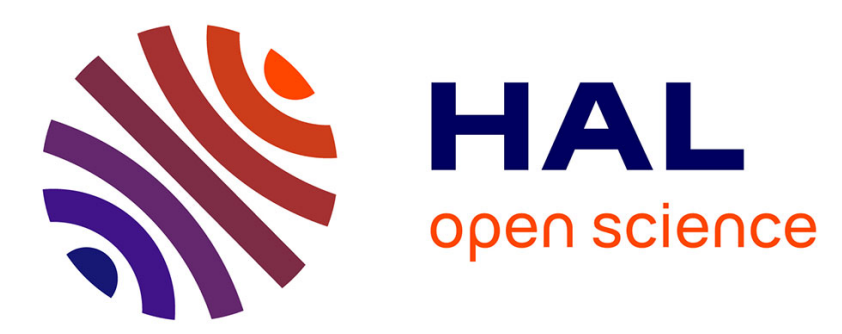

\title{
Vibrational analysis of structures with stochastic interfaces in the medium-frequency range: Experimental validation on a touch screen
}

Philippe Rouch, Claude Blanzé

\section{- To cite this version:}

Philippe Rouch, Claude Blanzé. Vibrational analysis of structures with stochastic interfaces in the medium-frequency range: Experimental validation on a touch screen. Journal of Sound and Vibration, 2014, 333 (6), pp.1612-1628. 10.1016/j.jsv.2013.11.014 . hal-03177136

\author{
HAL Id: hal-03177136 \\ https://hal.science/hal-03177136
}

Submitted on 6 May 2021

HAL is a multi-disciplinary open access archive for the deposit and dissemination of scientific research documents, whether they are published or not. The documents may come from teaching and research institutions in France or abroad, or from public or private research centers.
L'archive ouverte pluridisciplinaire HAL, est destinée au dépôt et à la diffusion de documents scientifiques de niveau recherche, publiés ou non, émanant des établissements d'enseignement et de recherche français ou étrangers, des laboratoires publics ou privés. 


\title{
Vibrational analysis of structures with stochastic interfaces in the medium-frequency range: Experimental validation on a touch screen
}

\author{
Philippe Rouch ${ }^{\mathrm{a}}$, Claude Blanzé ${ }^{\mathrm{b}}$ \\ a LBM, Arts et Métiers ParisTech, 151, boulevard de l'hôpital, 75013 Paris \\ ${ }^{\mathrm{b}}$ Structural Mechanics and Coupled Systems Laboratory, Cnam Paris, 2 rue Conté, 75003, Paris
}

\begin{abstract}
A B S T R A C T
This paper proposes a dedicated approach and its experimental validation when dealing with structures (including stochastic parameters, such as interface parameters) in mediumfrequency vibrations. The first ingredient is the use of a dedicated approach - the Variational Theory of Complex Rays (VTCR) - to solve the medium-frequency problem. The VTCR, which uses two-scale shape functions verifying the dynamic equation and the constitutive relation, can be viewed as a means of expressing the power balance at the different interfaces between substructures. The second ingredient is the use of the Polynomial Chaos Expansion (PCE) to calculate the random response. Since the only uncertain parameters are those which appear in the interface equations (which, in this application, are the complex connection stiffness parameters), this approach leads to very low computation costs. This method is validated on a new kind of touch screen. The simulated mobilities are compared with experimental ones obtained with a laser vibrometer and a good agreement is founded on a large medium-frequency bandwidth.
\end{abstract}

\section{Introduction}

Today, the main numerical modeling techniques for the analysis of medium-frequency vibrations [1] are most commonly based on the finite element method (FEM) or the boundary element method (BEM). In order to represent small-wavelength phenomena in complex structures (such as car chassis, satellites or ships), these techniques require huge numbers of degrees of freedom, since at least seven elements per wavelength are required to represent oscillating solutions properly [2]. In addition, the solutions obtained are highly sensitive to the material properties and boundary conditions. Moreover, damping is often localized at the structural connections; its measurement is very difficult and very error-prone. When we add to these difficulties, the will to take into account the variability inherent to mass production, the problem becomes very complex. In order to obtain the stochastic response of the structure, one must carry out a large number of costly computations. The use of high-frequency approaches, such as Statistical Energy Analysis (SEA) [3] or any of its improvements, appears unsuitable for medium-frequency vibrations: the vibrational behavior becomes too global and, in general, the coupling loss factor cannot be calculated in a predictive way. This paper proposes efficient techniques to obtain effective quantities when dealing with complex structures involving stochastic parameters, such as interface parameters. The first ingredient of these techniques is the use of a dedicated approach - the Variational Theory of Complex Rays (VTCR) [4] - to solve the medium-frequency problem. The VTCR, which uses two-scale shape functions verifying the dynamic equation and the constitutive relation, can be viewed as a mean of expressing the power balance at the different interfaces between substructures. The second ingredient is the use of the Polynomial Chaos Expansion (PCE) [5-9] to calculate the 
random response. Since the only uncertain parameters are those which appear in the interface equations (which, in this application, are the complex connection stiffness parameters), this approach leads to very low computation costs [10].

The application presented here concerns a new kind of touch screen (Fig. 1). Based on the recognition of vibrational signatures, this system is able to detect impact position in a very accurate way (accuracy: \pm 0.1 in, resolution 25 ppi) without the classical drawbacks of resistive or capacitive technologies.

The detection process uses two piezoelectric sensors that are connected to the glass that is clamped to its frame. They measure in real time the vibrational state of the rectangular plate and compare these signals to previously recorded signatures on a fine grid of the screen. A dedicated algorithm matches the signal with a signature included in the database and sends back to the system the impact point position.

In order to isolate the screen from parasite noise, the glass plate has its four edges sealed between two viscoelastic seals in a screen frame. As these seals have a stiffness and a damping factor that strongly depend on the compression ratio, the dynamic behavior of the touch screen can be greatly affected by the frame. The goal of this application is to simulate the influence of the seals properties in the screen dynamic signature and to compare the prediction with experimental data in the medium-frequency range (in this case $1.3 \mathrm{kHz}-6.5 \mathrm{kHz}$ ).

The paper will detail step by step the approach used for the simulation of the stochastic behavior of the screen: the model, the discretization, the polynomial chaos expansion, the parametric stochastic modeling, the numerical aspects and finally the experimental validation.

\section{Basic aspects of the VTCR}

\subsection{The reference problem}

Here, in order to simplify the presentation and to introduce the interface relations, the problem will be formulated for an assembly of only two substructures, but this can be easily generalized to an assembly of $n$ substructures. As an illustration,

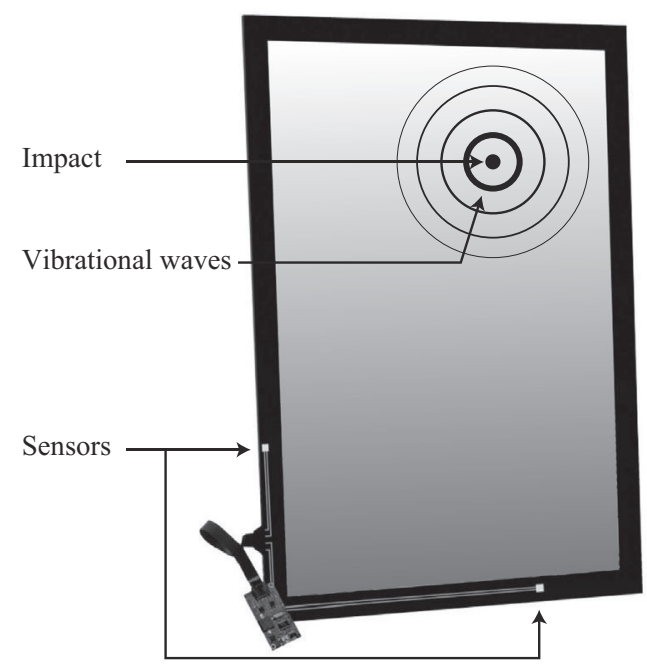

Fig. 1. New technology touch screen.

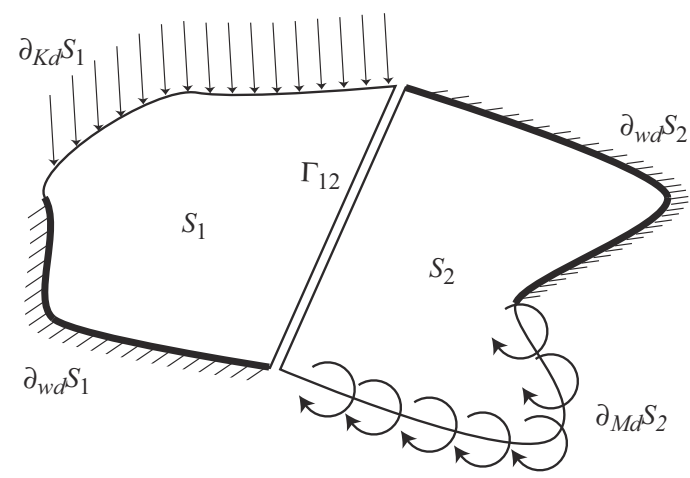

Fig. 2. Reference problem. 
let us consider two thin, homogeneous, isotropic and elastic Kirchhoff-Love plates and study the steady-state vibrations of this assembly. Classically, all quantities are defined in the complex domain and depend only on the space quantities $\mathbf{x}$ : any complex quantity $\mathbf{q}(\mathbf{x})$ is associated with $\mathbf{q}(\mathbf{x}, t)$ by the relation $\mathbf{q}(\mathbf{x}, t)=\mathbf{q}(\mathbf{x}) \cdot \exp (i \omega t)$ where $\omega$ is the fixed angular frequency and $t$ is the time.

Let $S_{1}$ and $S_{2}$ be two plates solicited harmonically at a fixed angular frequency $\omega, \partial S_{1}$ and $\partial S_{2}$ their respective boundaries, and $\Gamma_{12}$ the interface between $S_{1}$ and $S_{2}$. By considering that $l$ is the number of the studied plate, the boundary conditions are the following: prescribed deflection $w_{l}^{d}$ on part $\partial_{w_{d}} S_{l}$ of $\partial S_{l}$, prescribed slope $w_{n l}^{d}$ on $\partial_{w n_{d}} S_{l}$, prescribed bending moment $M_{l}^{d}$ on $\partial_{M_{d}} S_{l}$ and, finally, prescribed Kirchhoff shear $K_{l}^{d}$ on $\partial_{K_{d}} S_{l}$ (Fig. 2). The quantities of interest are the deflections $w_{l}$ and the moments $\mathbf{M}_{l}$.

For each plate $S_{l}$, let us introduce the admissible space $\mathcal{S}_{\text {ad }}^{l}$ of the displacement-moment pairs $S_{l}=\left(w_{l}, \mathbf{M}_{l}\right)$ defined on $S_{l}$ such that

$$
s_{l} \in \mathcal{S}_{\mathrm{ad}}^{l} \Leftrightarrow\left\{\begin{array}{c}
\left.w_{l} \in \mathcal{U} \quad \text { (set of finite-energy displacement field } H^{1}\left(S_{l}\right)\right) \\
\mathbf{M}_{l} \in \mathcal{S} \quad\left(\text { set of finite-energy moment field }\left[L^{2}\left(S_{l}\right)\right]^{3}\right) \\
\nabla^{2} \nabla^{2} w_{l}-k_{l}^{4} w_{l}=0 \quad \text { on } S_{l} \\
\mathbf{M}_{l}=\frac{2 h_{l}^{3}}{3}\left(1+\mathrm{i} \eta_{l}\right) \quad \mathbf{K}_{\mathrm{PS}_{l}} \mathcal{X}\left(w_{l}\right) \\
\text { for } \quad l=1,2 \\
\text { with } \quad k_{l}^{4}=\frac{3 \rho_{l} \omega^{2}\left(1-\nu_{l}^{2}\right)}{\left(1+\mathrm{i} \eta_{l}\right) E_{l} h_{l}^{2}}
\end{array}\right.
$$

where $k_{l}$ is the wavenumber and $\rho_{l}, \nu_{l}, \eta_{l}, E_{l}$ and $2 h_{l}$ designate respectively the density, Poisson's ratio, structural damping coefficient, Young's modulus and thickness of plate $S_{l}$. $\mathbf{K}_{\mathrm{PS}}$ is the Hooke's tensor for plane stress and $\mathcal{X}$ is the curvature operator. The fields of $\mathcal{S}_{\mathrm{ad}}^{l}$ are admissible in the sense that they verify both the local equilibrium and the constitutive relation exactly. The reference problem is

Problem 1. Find $s_{l}$ such that: $s_{l} \in \mathcal{S}_{\text {ad }}^{l}$

$$
\begin{aligned}
& \left\{\begin{array}{lll|lll}
w_{l}=w_{l}^{d} & \text { on } & \partial_{w_{d}} S_{l} & & & \\
w_{l, n_{l}}=w_{n_{l}}^{d} & \text { on } & \partial_{w n_{d}} S_{l} & w_{2} & \text { on } & \Gamma_{12} \\
M_{l n_{l}}=M_{l}^{d} & \text { on } & \partial_{M_{d}} S_{l} & w_{1, n_{1}}=w_{2, n_{2}} & \text { on } & \Gamma_{12} \\
K_{l n_{l}}=K_{l}^{d} & \text { on } & \partial_{K_{d}} S_{l} & K_{1 n_{1}}=K_{2 n_{2}} & \text { on } & \Gamma_{12} \\
\text { for } \quad l=1,2 & & & & \Gamma_{12}
\end{array}\right. \\
& \text { with } \quad M_{l n_{l}}=\mathbf{n}_{l} \cdot \mathbf{M}_{l} \mathbf{n}_{l} \text { and } K_{l n_{l}}=\mathbf{n}_{l} \cdot \operatorname{div}\left[\mathbf{M}_{l}\right]+\left(\mathbf{t}_{l} \cdot \mathbf{M}_{l} \mathbf{n}_{l}\right)_{, \mathbf{t}_{l}}
\end{aligned}
$$

where $\mathbf{n}_{l}$ and $\mathbf{t}_{l}$ are respectively the external normal and the tangent of each edge.

\subsection{Variational formulation associated with the VTCR}

The VTCR is primarily a global formulation of the boundary conditions and transmission conditions in terms of both displacements and forces. With this understanding, the problem becomes

Problem 2. Find $s_{l}$ such that

$$
\left\{\begin{array}{l}
s_{l} \in \mathcal{S}_{\mathrm{ad}}^{l} \\
\sum_{l}\left(A_{\partial S_{l}}\left(\delta s_{l}, s\right)-L_{\partial S}\left(\delta s_{l}\right)\right)+\sum_{\substack{l, m \\
l<m}} C_{\Gamma_{l m}}\left(\delta s_{l}, s_{l}, \delta s_{m}, s_{m}\right)=0 \\
\forall \delta s_{l} \in \mathcal{S}_{a d}^{l} \quad \text { for } \quad l=1,2 \quad m=1,2
\end{array}\right.
$$

with the boundary conditions:

$$
\begin{aligned}
A_{\partial S_{l}}\left(\delta s_{l}, S_{l}\right)-L_{\partial S_{l}}\left(\delta S_{l}\right) & =\operatorname{Re}\left\{-\mathrm{i} \omega\left(-\int_{\partial_{w_{d}} S_{l}} \delta \mathrm{K}_{l n_{l}}\left(\mathrm{w}_{l}-\mathrm{w}_{l}^{d}\right)^{*} \mathrm{dL}+\int_{\partial_{w n_{d}} S_{l}} \delta \mathbf{n}_{l} \cdot \mathbf{M}_{l} \mathbf{n}_{l}\left(w_{l, n_{l}}-w_{l, n_{l}}^{d}\right)^{*} \mathrm{~d} L\right.\right. \\
& \left.\left.+\int_{\partial_{M_{d}} S_{l}}\left(\mathbf{n}_{l} \cdot \mathbf{M}_{l} \mathbf{n}_{l}-M_{l}^{d}\right) \delta w_{l, n_{l}}^{*} \mathrm{~d} L-\int_{\partial_{K_{d}} S_{l}}\left(K_{l n_{l}}-K_{l}^{d}\right) \delta w_{l}^{*} \mathrm{~d} L\right)\right\}
\end{aligned}
$$


and with the transmission conditions on $\Gamma_{12}$ (for a perfect interface, continuity of the displacement and surfaces forces):

$$
\begin{aligned}
C_{\Gamma_{l m}}\left(\delta s_{l}, s_{l}, \delta s_{m}, s_{m}\right) & =\operatorname{Re}\left\{-\mathrm{i} \omega\left(\int _ { \Gamma _ { l m } } \frac { 1 } { 2 } \left[-\delta\left(\mathrm{K}_{l n_{l}}-\mathrm{K}_{m n_{m}}\right)\left(\mathrm{w}_{l}-\mathrm{w}_{m}\right)^{*}+\delta\left(\mathbf{n}_{l} \cdot \mathbf{M}_{l} \mathbf{n}_{l}+\mathbf{n}_{m} \cdot \mathbf{M}_{m} \mathbf{n}_{m}\right)\left(w_{l, \mathbf{n}_{l}}+w_{m, \mathbf{n}_{m}}\right)^{*}\right.\right.\right. \\
& \left.\left.\left.+\left(\mathbf{n}_{l} \cdot \mathbf{M}_{l} \mathbf{n}_{l}-\mathbf{n}_{m} \cdot \mathbf{M}_{m} \mathbf{n}_{m}\right) \delta\left(w_{l, \mathbf{n}_{l}}-w_{m, \mathbf{n}_{m}}\right)^{*}-\left(K_{l n_{l}}+K_{m n_{m}}\right) \delta\left(w_{l}+w_{m}\right)^{*}\right] \mathrm{~d} L\right)\right\}
\end{aligned}
$$

Re[.] and [.]* designate respectively the real part and the conjugate.

In practice, the VTCR procedure consists of two stages: first, one builds admissible fields; then, one introduces the boundary conditions through the discretization of the variational formulation associated with a set of admissible fields chosen among the elements of $\mathcal{S}_{a d}^{l}$.

\subsection{Construction of admissible fields}

Let us define a subset $\mathcal{S}_{a d}^{l h}$ of $\mathcal{S}_{a d}^{l}$. The VTCR uses two-scale approximations. In the vicinity of a point $\mathbf{x}$ of $S_{l}$, the displacement field is described as a superposition of rays which can be written as follows:

$$
W_{l}(\mathbf{x}, \mathbf{k})=\exp (\mathbf{k} . \mathbf{x}) a_{l}(\mathbf{k})=w_{e_{l}}(\mathbf{x}, \mathbf{k}) a_{l}(\mathbf{k})
$$

where $w_{e_{l}}(\mathbf{x}, \mathbf{k})$ represents the "fast" part of the field (it is defined explicitly) and $a_{l}(\mathbf{k})$ (that is the unknown)represents the ray amplitude which is a "slow" part. $\mathbf{k}$ denotes a vector which characterizes the local vibration ray and is calculated such that the ray verifies the dynamic equations. The moments are deduced from the constitutive relation:

$$
\mathbf{M}_{l}(\mathbf{x}, \mathbf{k})=\left(1+\mathrm{i} \eta_{l}\right) \mathbf{K}_{P S_{l}} \mathcal{X}\left(W_{l}(\mathbf{x}, \mathbf{k})\right)
$$

As the rays belong to the admissible space $\mathcal{S}_{\text {ad }}^{l}$, they must satisfy the admissibility relation

$$
\nabla^{2} \nabla^{2} W_{l}-k_{l}^{4} W_{l}=0 \text { on } S_{l}
$$

We have then:

$$
(\mathbf{k} . \mathbf{k})^{2}=k_{l}^{4}
$$

There are many possible such choices, which depend on the kind of rays that one would like to use for the solution approximation. The VTCR distinguishes the interior rays $W_{l}^{\text {int }}$, the edge rays $W_{l}^{\text {edg }}$ and the corner rays $W_{l}^{\text {cor }}$ :

$$
\begin{aligned}
& W_{l}^{\text {int }}(\mathbf{x}, \mathbf{k})=w_{e_{l}}^{\text {int }}(\mathbf{x}, \mathbf{k}) a_{l}^{\text {int }}(\mathbf{k}) \\
& W_{l}^{\text {edg }}(\mathbf{x}, \mathbf{k})=w_{e_{l}}^{\text {edg }}(\mathbf{x}, \mathbf{k}) a_{l}^{\text {edg }}(\mathbf{k}) \\
& W_{l}^{\text {cor }}(\mathbf{x}, \mathbf{k})=w_{e_{l}}^{\text {cor }}(\mathbf{x}, \mathbf{k}) a_{l}^{\text {cor }}(\mathbf{k})
\end{aligned}
$$

For example, if we would like to use interior rays, the locus of the end of the admissible vector $\mathbf{k}$ is a curve $\mathcal{C}_{l}^{\text {int }}$, depending on the material characteristics. For interior rays, this curve is a circle of radius $r$ (Fig. 3). Following the circular path, each direction of propagation within the plate is taken into account.
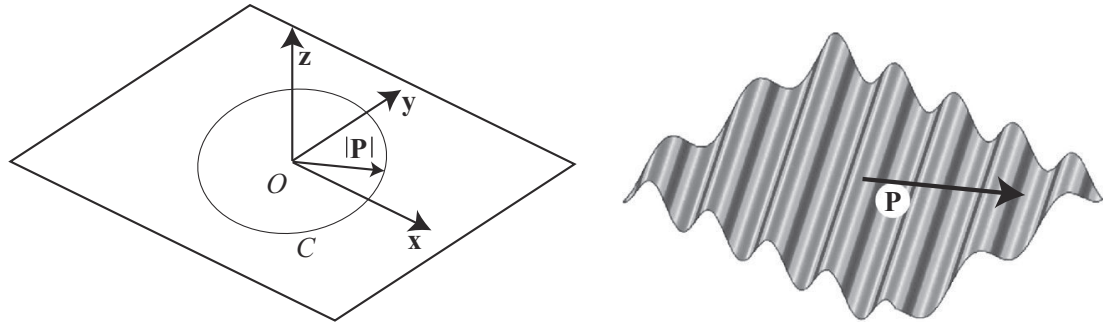

Fig. 3. Admissible vector for interior rays.
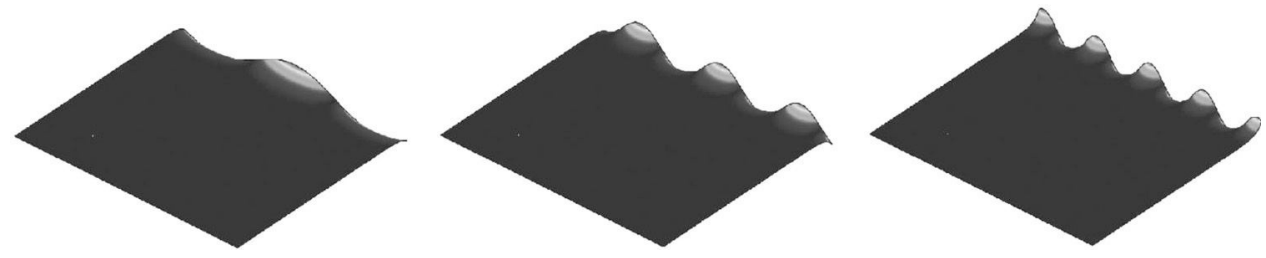

Fig. 4. Admissible edge rays for a plate. 

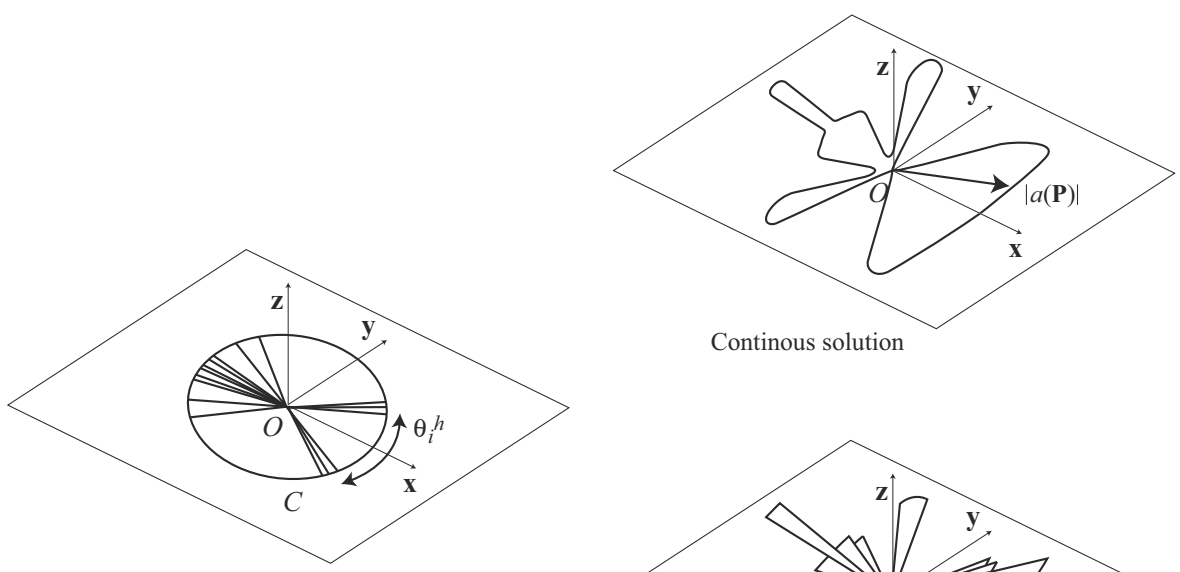

Discretized curve $C$

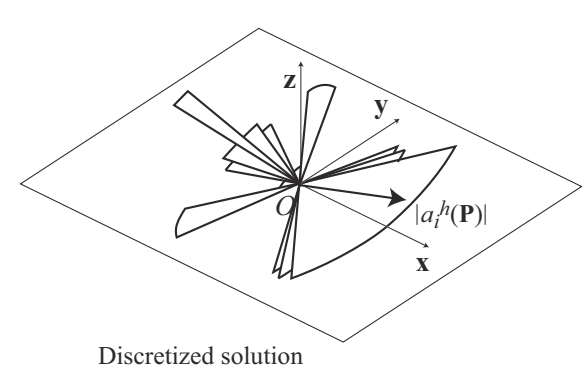

Fig. 5. Discretization and polar representation of continuous and discretized solution.

The definitions of corner and edge rays are very similar. The loci of the end of the admissible vector $\mathbf{k}$ are denoted as $\mathcal{C}_{l}^{\text {edg }}$ and $\mathcal{C}_{l}^{\text {cor }}$. Examples of edge rays can be seen in Fig. 4.

One of the advantages of the VTCR is to take into account all admissible ray directions. The solution $w_{l}^{\text {sol }}$ on the plate $l$ is then searched as

$$
\begin{gathered}
w_{l}^{\text {sol }}(\mathbf{x})=\int_{\mathcal{C}_{l}^{\text {int }}} a_{l}^{\text {int }}(\mathbf{k}) w_{e_{l}}^{\text {int }}(\mathbf{x}, \mathbf{k}) \mathrm{d} s+\int_{\mathcal{C}_{l}} a_{l}^{\text {edg }}(\mathbf{k}) w_{e_{l}}^{\text {edg }}(\mathbf{x}, \mathbf{k}) \mathrm{d} s \\
+\int_{\mathcal{C}_{l}^{\text {cor }}} a_{l}^{\text {cor }}(\mathbf{k}) w_{e_{l}}^{\text {cor }}(\mathbf{x}, \mathbf{k}) \mathrm{d} s
\end{gathered}
$$

\subsection{Discretized form of the VTCR}

In order to get a finite dimension problem, one discretizes the curves $\mathcal{C}_{l}^{\text {int }}, \mathcal{C}_{l}^{\text {edg }}$ and $\mathcal{C}_{l}^{\text {cor }}$ into a finite number of elements which can be of different sizes. For example, in Fig. $5(\mathrm{~b}), \mathcal{C}_{l}^{\text {int }}$ is discretized into 16 elements of variable size in order to describe with the smallest number of degrees of freedom the continuous solution Fig. 5(a). The amplitude $a_{l}^{\text {int }}(\mathbf{k})$ is considered to be constant along angular sectors $\theta_{i_{i}}^{\text {int }}$ and is denoted $a_{i_{1}}^{\text {int }}$ as represented in Fig. 5(c).

Then the discretized solution $\mathrm{w}_{l}^{h}$ in the plate $l$ is defined by

$$
\begin{aligned}
w_{l}^{h}(\mathbf{x})=\sum_{i=1}^{n} a_{i_{l}}^{\text {int }} & \int_{\theta_{i_{l}}} w_{e_{l}}^{\text {int }}(\mathbf{x}, \mathbf{k}) \mathrm{d} s+\sum_{i=1}^{m} a_{i_{l}}^{\text {edg }} \int_{\theta_{i_{l}}^{\text {edg }}} w_{e_{l}}^{\text {edg }}(\mathbf{x}, \mathbf{k}) \mathrm{d} s \\
& +\sum_{i=1}^{p} a_{i_{l}}^{\text {cor }} \int_{\theta_{i_{l}}^{\text {cor }}} w_{e_{l}}^{\text {cor }}(\mathbf{x}, \mathbf{k}) \mathrm{d} s \\
= & \sum_{i=1}^{n} a_{i_{l}}^{\text {int }} w_{i_{l}}^{\text {int }}(\mathbf{x})+\sum_{i=1}^{m} a_{i_{l}}^{\text {edg }} w_{i_{l}}^{\text {edg }}(\mathbf{x})+\sum_{i=1}^{p} a_{i_{l}}^{\text {cor }} w_{i_{l}}^{\text {cor }}(\mathbf{x})
\end{aligned}
$$

where the generalized amplitude $a_{i_{\perp}}^{\text {int }}$ is associated with the basis function $w_{i_{1}}^{\text {int }}(\mathbf{x})$, corresponding to the integral of an elementary interior ray over an angular sector $\theta_{i_{l}}^{\text {int }}$ related to $\mathcal{C}_{l}^{\text {int }}$ (equivalent writings are defined for the edge and the corner rays).

Once discretized, the VTCR formulation leads to a system of linear equations of finite dimension in the complex domain:

$$
\mathbf{c a}=\mathbf{f}
$$

where $\mathbf{c}$ is the coupling matrix, $\mathbf{a}$ is the generalized amplitudes vector and $\mathbf{f}$ is the generalized forces vector.

\section{Modeling of the interfaces}

In industrial structures, most of the damping is localized in the different joints connecting the substructures [11,12]. These joints, whether welded, riveted, bolted or glued modify the structure's response. Since these joints are usually 

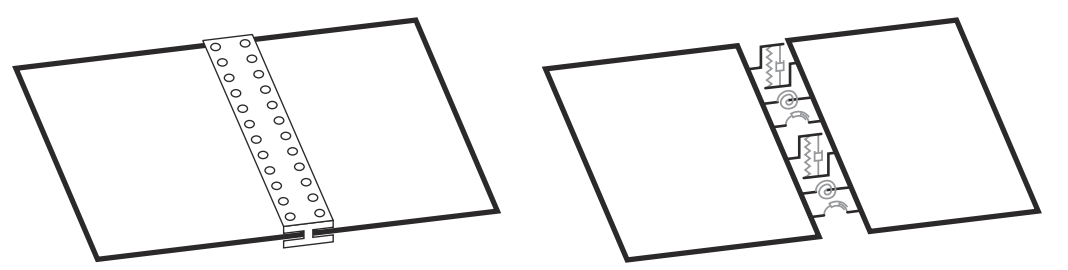

Fig. 6. Joint between two plates.

distributed along the edges of the sub-structures, we propose to introduce them by using distributed interface parameters. The generic joint behavior is then modeled by interface relations depending on elastic and/or dissipative joint parameters represented by a complex stiffness. Two main cases can occur: joint between two substructures or joint between a substructure and the frame.

\subsection{Joints between two substructures}

If two plates are connected in the same plane by interface $\Gamma_{12}$ as presented in Fig. 6 , the relations governing the coupling can be expressed using two types of equations:

- the equilibrium of the interface

$$
\begin{gathered}
-\mathbf{n}_{1} \cdot \mathbf{M}_{1} \mathbf{n}_{1}+\mathbf{n}_{2} \cdot \mathbf{M}_{2} \mathbf{n}_{2}=0 \\
K_{1 n_{1}}+K_{2 n_{2}}=0
\end{gathered}
$$

- the constitutive relations governing the interface

$$
\begin{gathered}
\frac{1}{2}\left(\mathbf{n}_{1} \cdot \mathbf{M}_{1} \mathbf{n}_{1}+\mathbf{n}_{2} \cdot \mathbf{M}_{2} \mathbf{n}_{2}\right)=k_{b}\left(1+\mathrm{i} \eta_{b}\right)\left(w_{1, n_{1}}+w_{2, n_{2}}\right) \\
\frac{1}{2}\left(K_{1 n_{1}}-K_{2 n_{2}}\right)=k_{t}\left(1+\mathrm{i} \eta_{t}\right)\left(w_{1}-w_{2}\right)
\end{gathered}
$$

where $k_{r}$ and $\eta_{r}$ are the stiffness and the damping associated to the complex rotational spring while $k_{t}$ and $\eta_{t}$ are the stiffness and the damping associated to the complex translational spring.

The variational form on interface $\Gamma_{12}$ of these conditions is given by

$$
\begin{gathered}
C_{\Gamma_{12}}=\operatorname{Re}\left\{-\mathrm{i} \omega\left(-\int_{\Gamma_{12}} \frac{1}{2} \delta\left[\left(\mathrm{K}_{1 n_{1}}-\mathrm{K}_{2 n_{2}}\right)-\mathrm{k}_{t}\left(1+\mathrm{i} \eta_{t}\right)\left(\mathrm{w}_{1}-\mathrm{w}_{2}\right)\right]\left(\mathrm{w}_{1}-\mathrm{w}_{2}\right)^{*} \mathrm{dL}\right.\right. \\
-\int_{\Gamma_{12}} \frac{1}{2}\left(K_{1 n_{1}}+K_{2 n_{2}}\right) \delta\left(w_{1}+w_{2}\right)^{*} \mathrm{~d} L \\
+\int_{\Gamma_{12}} \frac{1}{2} \delta\left[\left(\mathbf{n}_{1} \cdot \mathbf{M}_{1} \mathbf{n}_{1}+\mathbf{n}_{2} \cdot \mathbf{M}_{2} \mathbf{n}_{2}\right)-k_{b}\left(1+\mathrm{i} \eta_{b}\right)\left(w_{1, n_{1}}+w_{2, n_{2}}\right)\right]\left(w_{1, n_{1}}+w_{2, n_{2}}\right)^{*} \mathrm{~d} L \\
\left.\left.+\int_{\Gamma_{12}} \frac{1}{2}\left(\mathbf{n}_{1} \cdot \mathbf{M}_{1} \mathbf{n}_{1}-\mathbf{n}_{2} \cdot \mathbf{M}_{2} \mathbf{n}_{2}\right) \delta\left(w_{1, n_{1}}-w_{2, n_{2}}\right)^{*} \mathrm{~d} L\right)\right\}
\end{gathered}
$$

This term replaces the perfect interface formulation part expressed at Eq. (4).

\subsection{Joints between a substructure and the frame}

If a substructure is connected to the frame via a joint as presented in Fig. 7, the relations governing the coupling are given by two local constitutive relations:

$$
\begin{gathered}
\mathbf{n}_{l} \cdot \mathbf{M}_{l} \mathbf{n}_{l}-k_{r}\left(1+\mathrm{i} \eta_{r}\right) w_{l, n_{l}}=0 \\
K_{l n_{l}}-k_{t}\left(1+\mathrm{i} \eta_{t}\right) w_{l}=0
\end{gathered}
$$



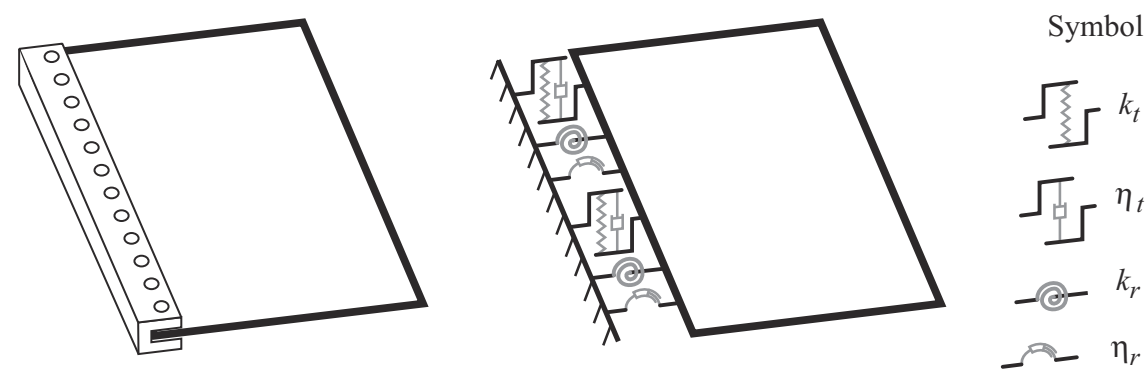

Fig. 7. Joint between a plate and its frame.

The variational form on the corresponding edge $\partial_{3} S_{l}$ of these conditions is given by

$$
\begin{aligned}
A_{\partial_{3} S_{l}}-L_{\partial_{3} S_{l}}=\operatorname{Re} & \left\{-\mathrm{i} \omega\left(\int_{\partial_{3} S_{l}}\left(\mathbf{n}_{l} \cdot \mathbf{M}_{l} \mathbf{n}_{l}-\mathrm{k}_{r}\left(1+\mathrm{i} \eta_{r}\right) \mathrm{w}_{l, n_{l}}\right) \delta \mathrm{w}_{l, n_{l}}^{*} \mathrm{dL}\right)\right. \\
& \left.\left.-\int_{\partial_{3} S_{l}}\left(K_{l n_{l}}-k_{t}\left(1+\mathrm{i} \eta_{t}\right) w_{l}\right) \delta w_{l}^{*} \mathrm{~d} L\right)\right\}
\end{aligned}
$$

This term replaces the part of at Eq. (4) corresponding to $\partial_{3} S_{l}$ edge.

\section{Discretization in random space}

The efficient propagation of uncertainty from model parameters to model predictions (forward problem) is a central challenge of uncertainty quantification. A simple approach is Monte Carlo simulation: sampling known distributions of the model parameters to obtain statistics or density estimates of the model predictions. Again, each sample requires a solution of the forward model, and with complex models, this sampling approach is computationally intensive.

Perturbation approach or Neumann expansion is other useful way of quantifying the structural response uncertainty. In perturbation methods, the random fields are expanded via Taylor series around the mean values [13]. Neumann expansion has been used by some researchers instead of Taylor series for manipulation of the stochastic system properties and response [14]. The main inherent limitation of these methods is that the uncertainties cannot be large, i.e. the deviations of structure response cannot be too large compared with the nominal values.

A useful alternative is to employ spectral representations of uncertain parameters and field quantities, specifically polynomial chaos expansions (PCEs) for random variables and stochastic processes. The polynomial chaos [5] was first defined by Wiener [15]; successive polynomial chaoses give rise to a functional basis consisting of Hermite polynomials of Gaussian random variables [16]. Ghanem et al. [17] describe the implementation of polynomial chaos in a finite element context. These stochastic finite element approaches have found numerous modeling applications, including solid or structural mechanics $[18,6,9]$. Xiu et al. used generalized polynomial chaos for uncertainty quantification in fluid-structure interactions and in diffusion problems [19].

\subsection{Polynomial chaos expansion (PCE)}

The discretization of random variables by projection on polynomial chaos is recalled next. Let $(\Omega, \mathcal{F}, P)$ be a probability space, where $\Omega$ is a simple space, $\mathcal{F}$ is a $\sigma$-algebra over $\Omega$, and $P$ is a probability measure on $\mathcal{F}$. Also, let $\left\{\xi_{i}(\theta)\right\}_{i=1}^{\infty}$ be a set of orthonormal standard Gaussian random variables on $\Omega$. Any square-integrable random variable $X: \Omega \rightarrow \mathbb{R}$ has the following representation:

$$
\begin{aligned}
X(\theta)= & a_{0} \Gamma_{0}+\sum_{i=1}^{\infty} a_{i} \Gamma_{1}\left(\xi_{i}\right)+\sum_{i=1}^{\infty} \sum_{j=1}^{i} a_{i j} \Gamma_{2}\left(\xi_{i}, \xi_{j}\right) \\
& +\sum_{i=1}^{\infty} \sum_{j=1}^{i} \sum_{k=1}^{j} a_{i j k} \Gamma_{3}\left(\xi_{i}, \xi_{j}, \xi_{k}\right)+\cdots
\end{aligned}
$$

where $\Gamma_{p}$ is the Wiener polynomial chaos of order $p$. This expansion may be re-written in a more compact form:

$$
X(\theta)=\sum_{i=0}^{\infty} x_{i} \Psi_{i}(\theta)
$$

where there is a one-to-one correspondence between the coefficients and functionals in Eqs. (22) and (23). For the standard normal random variable $\xi$ chosen above, orthogonality of successive $\Gamma_{p}$ requires that the $\Gamma_{p}$ be multivariate Hermite polynomials; both these and the corresponding $\psi_{k}$ may be generated from univariate Hermite polynomials by taking tensor products. 
In computations, one truncates the expansion both of order $p$ and in dimension $n$ by choosing a finite subset $\{\xi\}=\left\{\xi_{\alpha_{i}}\right\}_{\alpha_{i}=1}^{n}$ of the infinite set $\left\{\xi_{i}\right\}_{i=1}^{\infty}, \alpha_{i} \in \mathbb{N}$. The total number of terms $P$ in the finite polynomial chaos expansion

$$
X_{p}(\theta)=\sum_{i=0}^{P} x_{i} \Psi_{i}(\theta)
$$

is

$$
P+1=\frac{(n+p) !}{n ! p !}
$$

Polynomial chaos expansions (PCEs) have been generalized to broader classes of orthogonal polynomials in the Askey scheme, each family resulting from a different choice of distribution for the $\xi_{\mathrm{i}}$ [19]. For each of these choices, orthogonality of the polynomials $\psi_{k}(\xi)$ with respect to the inner product on $L^{2}(\Omega)$ is guaranteed:

$$
\begin{gathered}
\left\langle\psi_{i} \psi_{j}\right\rangle=\int \psi_{i}(\xi(\theta)) \psi_{j}(\xi(\theta)) \mathrm{d} P(\theta)=\int \psi_{i}(\xi(\theta)) \psi_{j}(\xi(\theta)) w(\xi) \mathrm{d} \xi \\
=\delta_{i j}\left\langle\psi_{i}^{2}\right\rangle
\end{gathered}
$$

where, in the second integral, $w(\xi)$ denotes the probability density of $\xi$. This property can be used to calculate the truncated PC representation of a random variable $g \in L^{2}(\Omega)$ by projecting onto the PC basis:

$$
\tilde{g}(\theta)=\sum_{k=0}^{P} g_{k} \psi_{k}(\xi), \quad g_{k}=\frac{\left\langle g(X) \psi_{k}\right\rangle}{\left\langle\psi_{k}^{2}\right\rangle}
$$

This orthogonal projection minimizes the error $\|g-\tilde{g}\|$ on the space spanned by $\left\{\psi_{k}\right\}_{k=0}^{P}$ where $\| \bullet$ is the inner-product norm on $L^{2}(\Omega)$.

The stochastic interface parameters $\alpha(\theta)$ lead to a discretized linear system similar to Eq. (13):

$$
\mathbf{k}(\alpha(\theta)) \mathbf{a}(\theta)=\mathbf{f}
$$

where $\mathbf{a}(\theta)$ is the unknown generalized variables and $f$ is the environment load considered deterministic for simplicity's sake $^{1}$.

$\mathbf{a}(\theta)$ can be expressed formally as a nonlinear functional of the set $\left\{\xi_{j}(\theta)\right\}$ used to represent the material's stochastic property. As it has been noted previously [16], this functional dependence can be expanded in terms of polynomial chaos. Then, the truncated PCE of the response takes the form:

$$
\mathbf{a}(\theta)=\sum_{i=0}^{P} \mathbf{a}_{i} \Psi_{i}(\theta)
$$

where $\left\{\Psi_{i}(\theta)\right\}$ are polynomials in the Gaussian random variables $\left\{\xi_{i}\right\}$. The number of polynomials $P$ depends on the order $p$ of the PCE and on the number $n$ of stochastic parameters. Let us assume that the material parameters are constant along an interface. If this were not the case, a Karhunen-Loeve expansion could be used to represent the spatial randomness of the interface's characteristics [17].

\subsection{Parametric stochastic modeling}

Stochastic modeling consists in constructing (as objectively as possible) the probability law of the input parameters $\alpha(\theta)$ (for example the probability density function (pdf)). Some available information has to be taken into account such as:

- $\alpha(\theta)$ has to be a positive-valued random variable

- $\alpha(\theta)$ has to be a second-order random variable: $E\left(\alpha^{2}(\theta)\right)<\infty$

- the mean value is given: $E(\alpha(\theta))=\bar{\alpha}>0$

- the solution $\mathbf{a}(\theta)$ has to be a second-order random variable

When no other information is given (no experimental data for example) the pdf could be constructed using the maximum entropy principle [20]. For example, for a given bounded support $[a, b]$, the maximum entropy principle yields to a uniform random variable.

In this study, we want to represent the input parameters on the Wiener Chaos expansion. Therefore we want to express each parameter as a function of the set $\left\{\xi_{j}(\theta)\right\}$. Although the well-known Box-Muller transformation express a uniform random value $U$ as a nonlinear function of two Gaussian random variables $\left\{\xi_{1}, \xi_{2}\right\}$ such as

$$
U=\arctan \frac{\xi_{1}}{\xi_{2}}
$$

\footnotetext{
${ }^{1}$ If the environment load $f$ is random or uncertain, it has to be modeled by a random vector: this case is usual and without special difficulties
} 
the number of $\xi_{\mathrm{i}}$ needed is twice the number of parameters. So we choose to represent each stochastic input parameter $\alpha(\theta)$ by a nonlinear function of a single gaussian random variable:

$$
\alpha(\theta)=\bar{\alpha}(1+\delta g(\xi(\theta))) \text { with } g(x)=\frac{2 \arcsin \left(\operatorname{Erf}\left(\frac{x}{\sqrt{2}}\right)\right)}{\sqrt{-8+\pi^{2}}}
$$

where $\bar{\alpha}$ is the mathematical expectation of $\alpha(\theta), \delta$ is the coefficient of variation (standard deviation divided by the expectation) and $\xi(\theta)$ is a standard normal random variable: $\xi(\theta) \sim N(0,1)$ and $g$ is a nonlinear function so as to have a compact support for the probability density function of $\alpha(\theta)$ as presented in Fig. 8. This transformation ensures to have a positive values for the damping and the stiffness random variables.

In our application, the mean of the translational and rotational stiffnesses $\left(k_{t}\right.$ and $\left.k_{r}\right)$ and the translational and rotational loss factors $\left(\eta_{t}\right.$ and $\left.\eta_{r}\right)$ will be the ones identified in the ten frequencies steps. Regarding only the translational stiffness for sake of simplicity, its representation is a frequency dependent random variable as represented in Fig. 9.

\subsection{Assembling procedure}

Following the assembly procedure, this leads to the corresponding expansion of the matrix $\mathbf{K}(\theta)$ :

$$
\mathbf{K}(\alpha(\theta))=\mathbf{K}_{0}+\sum_{i=1}^{n} g\left(\xi_{i}(\theta)\right) \mathbf{k}_{i}
$$

where $\mathbf{K}_{\mathbf{0}}$ denotes the matrix corresponding to the mean material properties and the other terms are related to the random fluctuations about this mean. The number of stochastic parameters is $n$. Expanding the generalized parameters $\mathbf{a}(\theta)$ with respect to the polynomial chaos basis and substituting Eqs. (31) and (29) into Eq. (28) leads to

$$
\sum_{j=0}^{P} \Psi_{j}(\theta) \mathbf{k}_{0} \mathbf{a}_{j}+\sum_{j=0}^{P} \sum_{i=1}^{n} g\left(\xi_{i}\right) \Psi_{j}(\theta) \mathbf{k}_{i} \mathbf{a}_{j}=\mathbf{f}
$$

An equality (in a weak sense) can be derived by projecting Eq. (32) onto the subspace spanned by the polynomial chaos subset used in the approximation; this process results in the following equations where $\langle\bullet\rangle$ represent the mathematical

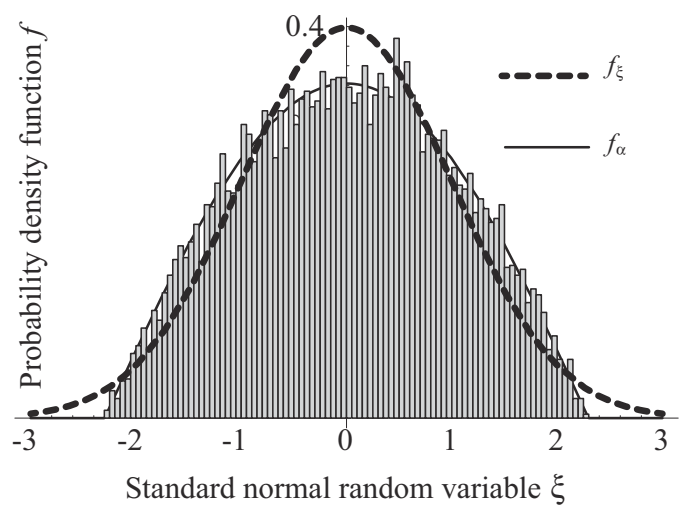

Fig. 8. Comparison between $\alpha$ 's and $\xi$ 's PDF.

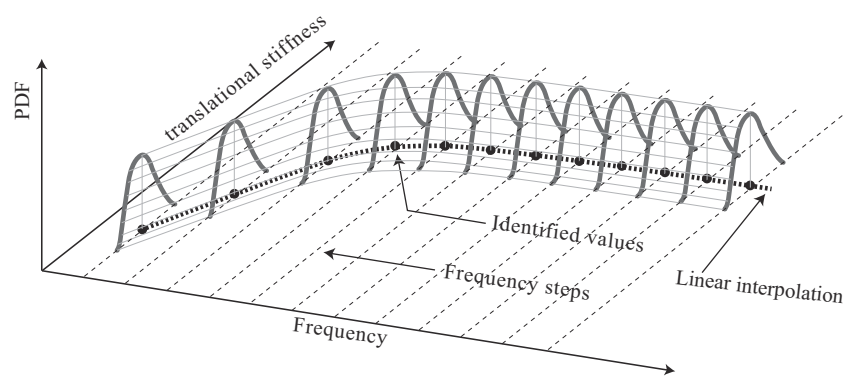

Fig. 9. Random translational stiffness. 
expectation:

$$
\sum_{j=0}^{P}\left(\left\langle\Psi_{j}(\theta) \Psi_{k}(\theta)\right\rangle \mathbf{k}_{0}+\sum_{i=1}^{n}\left\langle g\left(\xi_{i}\right) \Psi_{j}(\theta) \Psi_{k}(\theta)\right\rangle \mathbf{k}_{i}\right) \mathbf{a}_{j}=\left\langle\Psi_{k}(\theta)\right\rangle \mathbf{f} \quad k=0,1, \ldots P,
$$

This system can be rewritten as

$$
\sum_{j=0}^{P} \sum_{i=0}^{n} c_{i j k} \mathbf{k}_{i} \mathbf{a}_{j}=\delta_{0 k} \mathbf{f} \quad k=0,1, \ldots P,
$$

where the coefficients $c_{0 j k}$ denote $\delta_{j k}\left\langle\Psi_{j}(\theta) \Psi_{k}(\theta)\right\rangle$ and coefficients $c_{i j k}$ denote $\left\langle g\left(\xi_{i}\right) \Psi_{j}(\theta) \Psi_{k}(\theta)\right\rangle$ for $i>0$. They need to be calculated only once. This system of linear equations must be solved for the unknown $\mathbf{a}_{j}$ of the PCE. The details of this procedure were published in [5] and its implementation issues addressed in a number of other references [17,18]. These equations can be assembled into a matrix of size $(P+1) n \times(P+1) n(n$ being the number of degrees of freedom) of the form:

$$
\left(\begin{array}{ccccc}
\mathbf{k}^{(00)} & \cdot & \mathbf{k}^{(0 k)} & \cdot & \mathbf{k}^{(0 P)} \\
\cdot & \cdot & \cdot & \cdot & \cdot \\
\mathbf{k}^{(j 0)} & \cdot & \mathbf{k}^{(j k)} & \cdot & \mathbf{k}^{(j P)} \\
\cdot & \cdot & \cdot & \cdot & \cdot \\
\mathbf{k}^{(P 0)} & \cdot & \mathbf{k}^{(P k)} & \cdot & \mathbf{k}^{(P P)}
\end{array}\right)\left(\begin{array}{c}
\mathbf{a}_{0} \\
\cdot \\
\mathbf{a}_{k} \\
\cdot \\
\mathbf{a}_{P}
\end{array}\right)=\left(\begin{array}{c}
\mathbf{f} \\
\cdot \\
0 \\
\cdot \\
0
\end{array}\right)
$$

where

$$
\mathbf{k}^{(j k)}=\sum_{i=0}^{n} c_{i j k} \mathbf{k}_{i}
$$

\subsection{Resolution: numerical strategy}

The new linear problem related to Eq. (35) can be written as

$$
\tilde{\mathbf{K}} \tilde{\mathbf{A}}=\tilde{\mathbf{F}}
$$

$\tilde{\mathbf{K}}$ is a symmetric block matrix even if $\tilde{\mathbf{K}}^{(j k)}$ are nonHermitian matrices. Its structure is sparse and its dimension is usually rather large as explained above. In our application, as the initial size of a VTCR problem is very small ( $n$ is very small), the dimension of $\tilde{\mathbf{K}}$ is very acceptable by classic linear solvers. Despite everything and regarding its particular structure, we have used the iterative solving method GEMRES [21]. With this solving method, we have used a preconditioner P:

$$
\mathbf{P}=\left(\begin{array}{cccc}
\mathbf{k}_{0} & & & \\
& \cdot & & \\
& d_{j} \mathbf{k}_{0} & & \\
& & \cdot & \\
& & & d_{P} \mathbf{k}_{0}
\end{array}\right)^{-1} \text { with } d_{j}=\left\langle\Psi_{j}(\theta)^{2}\right\rangle
$$

The method has several advantages:

- it does not invert the global system (only $\mathbf{k}_{0}$ is inverted).

- it allows a large gain in memory (it only stores $\mathbf{k}_{0}^{-1}, \mathbf{k}_{i}, c_{i j k}$ and $\left.\left\langle\Psi_{j}(\theta)\right)^{2}\right\rangle$ ).

- it only use MATVEC products.

In summary, this approach consists in expanding the random response process about a basis of the Hilbert space of random variables and calculating the coefficients of this expansion. The result is a convergent expansion of the response in terms of multidimensional orthogonal polynomials. Although the methodology used is becoming widespread, serious obstacles have been encountered from a computational point of view in practical implementations. In large realistic problems, the methodology is either cumbersome or computationally intensive. Some numerical strategies, such as iterative algorithms, have been devised to overcome the numerical difficulties which arise in this context [22]. Our proposed approach does not present such drawbacks: it uses very small elementary matrices, thus leading to very small problems, and since the evolution of the generalized amplitude is relatively smooth it can be easily represented by the Hermitian polynomials of the chaos expansion.

\section{Experimental validation on a the touch screen}

The goal of this application is to demonstrate the ability of the VTCR to compute stochastic frequency response function and to compare this simulation with experimental data in the medium-frequency range. According to the definition of $[23,24]$ using the Modal Overlap count (MO) this bandwidth is between $1.3 \mathrm{kHz}$ and $6.5 \mathrm{kHz}$. As it is impossible to test 
enough different touch screens in order to generate the experimental variability, we will build a setup allowing us to have a wide range of clamping conditions between a single screen and its frame and we will make the assumption that this variability range is representative of the one that could be observed on a real industrial production. The approach will be decomposed in three steps:

- we will identify the joint properties on a calibrated setup.

- we will use this values as mean values for the numerical probabilistic model.

- we will then compare the numerical variability domain with the experimental one obtained by modifying the clamping conditions on the setup.

\subsection{Problem modeling}

We are modeling a 17 inch touch screen presented in Fig. 10. The characteristic dimensions of the glass are given by: $a=320 \mathrm{~mm}, b=245 \mathrm{~mm}, h=2.85 \mathrm{~mm}$. The glass structural parameters will be the one provided in [25]: $E=64 \mathrm{GPa}, \nu=0.22$ $\rho=2520 \mathrm{~kg} \mathrm{~m}^{-3}$.
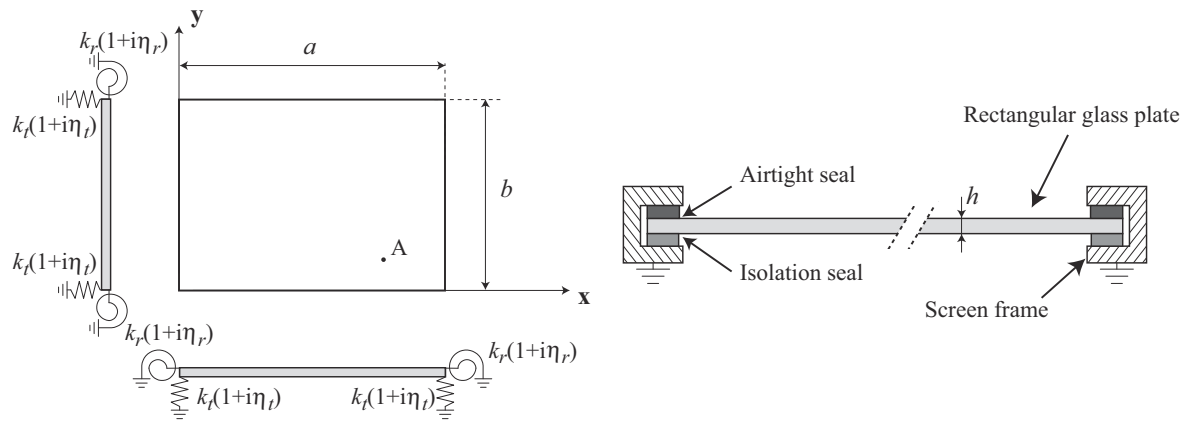

Fig. 10. Touch screen boundary conditions modeling.

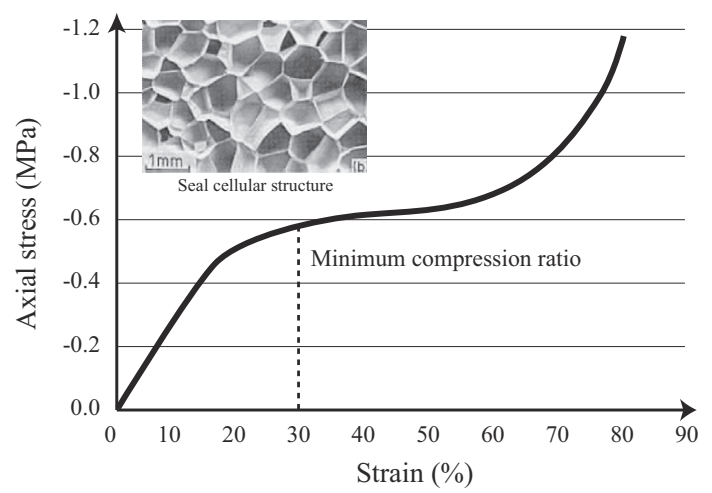

Fig. 11. Seals behavior for low strain rate.
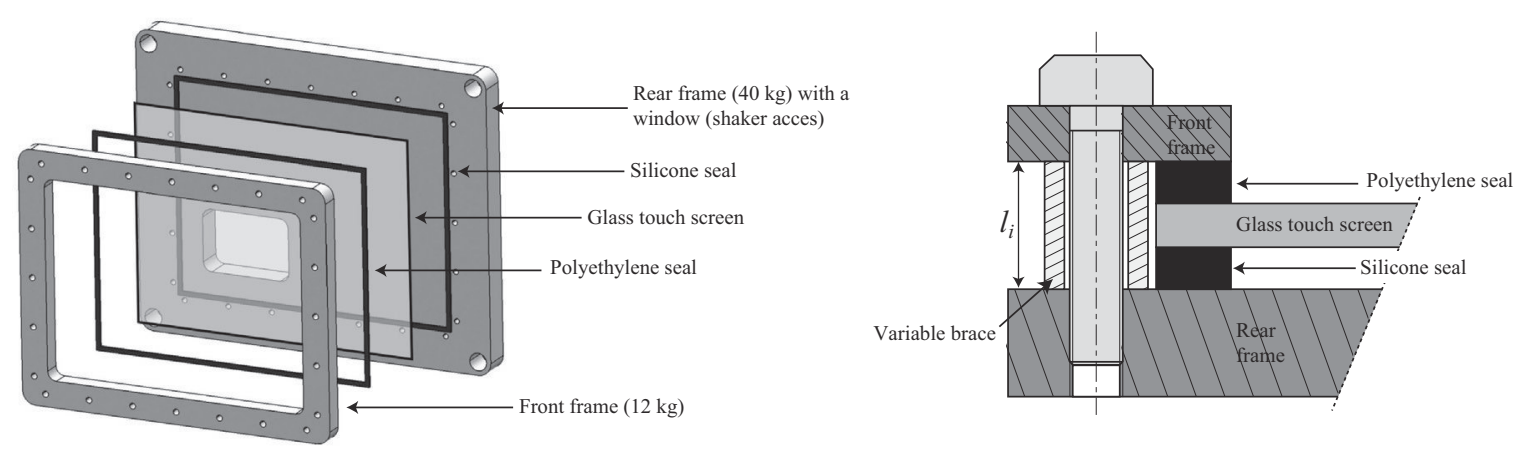

Fig. 12. Experimental setup including variable distance $l_{i}$ between the two frames. 
The screen is clamped in its frame using two viscoelastic seals (airtight seal and isolation seal). They isolate the glass plate and provide the largest part of the dissipation [26]. As the seals width is negligible compared to the glass plate characteristic dimensions, we have classically [27] modeled the two seals ensemble by a lineic complex spring (as described in chapter 3.2): $k_{r}$ and $\eta_{r}$ are the stiffness and the damping associated to the complex rotational spring while $k_{t}$ and $\eta_{t}$ are the stiffness and the damping associated to the complex translational spring. The values of the stiffnesses and the damping coefficients are highly dependent on the seal compression ratio and on the frequency as these seals have a nonlinear viscoelastic behavior associated with their cellular structure (Fig. 11).

For sealing reasons, the manufacturer imposes a minimum compression ratio of 30 percent: the seals work then in a pre-stress state where a weak variation of compression can generate a great variation of stiffness and damping. The values of nominal stiffness and damping will then be first identified for the recommended compression ratio (in our case 36 percent).
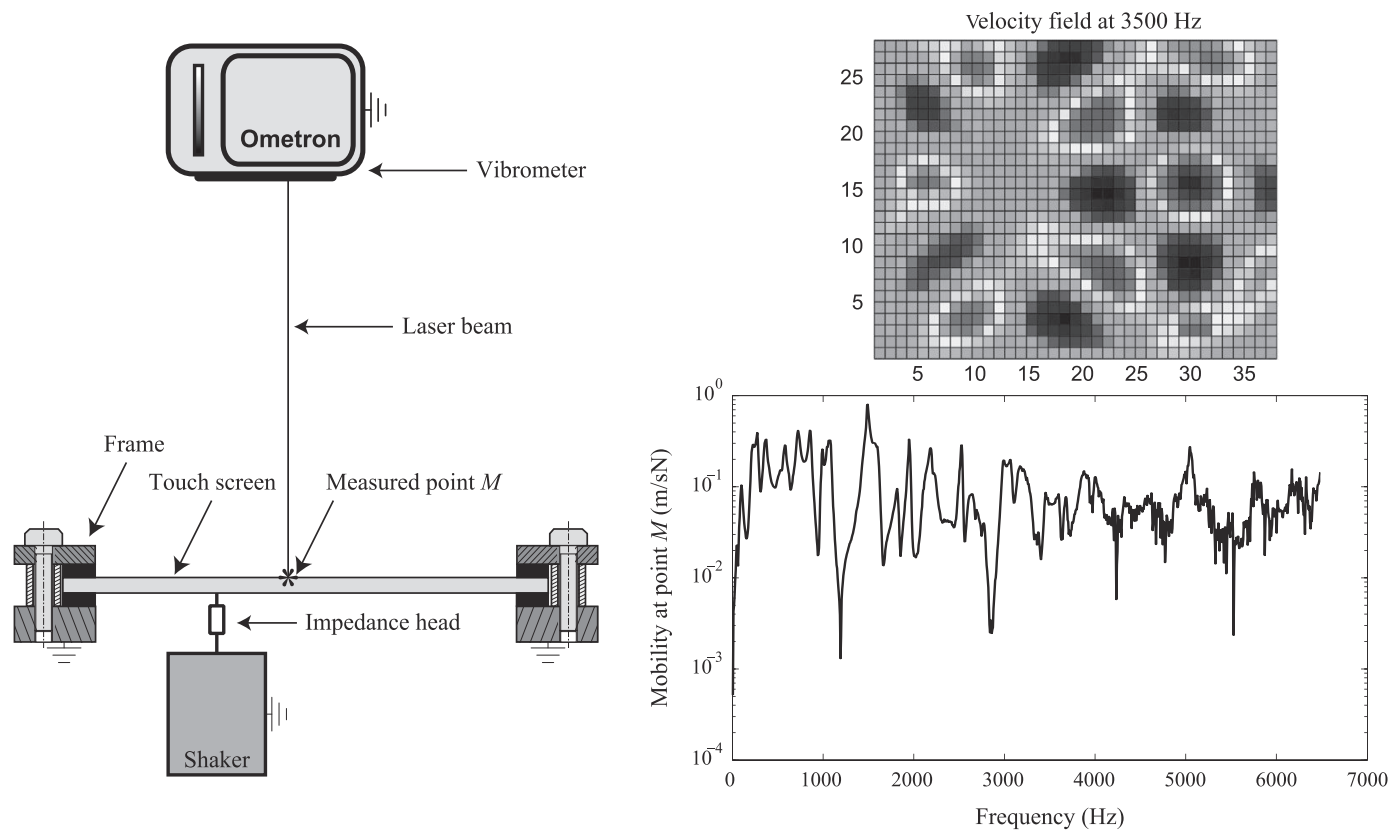

Fig. 13. Experimental setup.

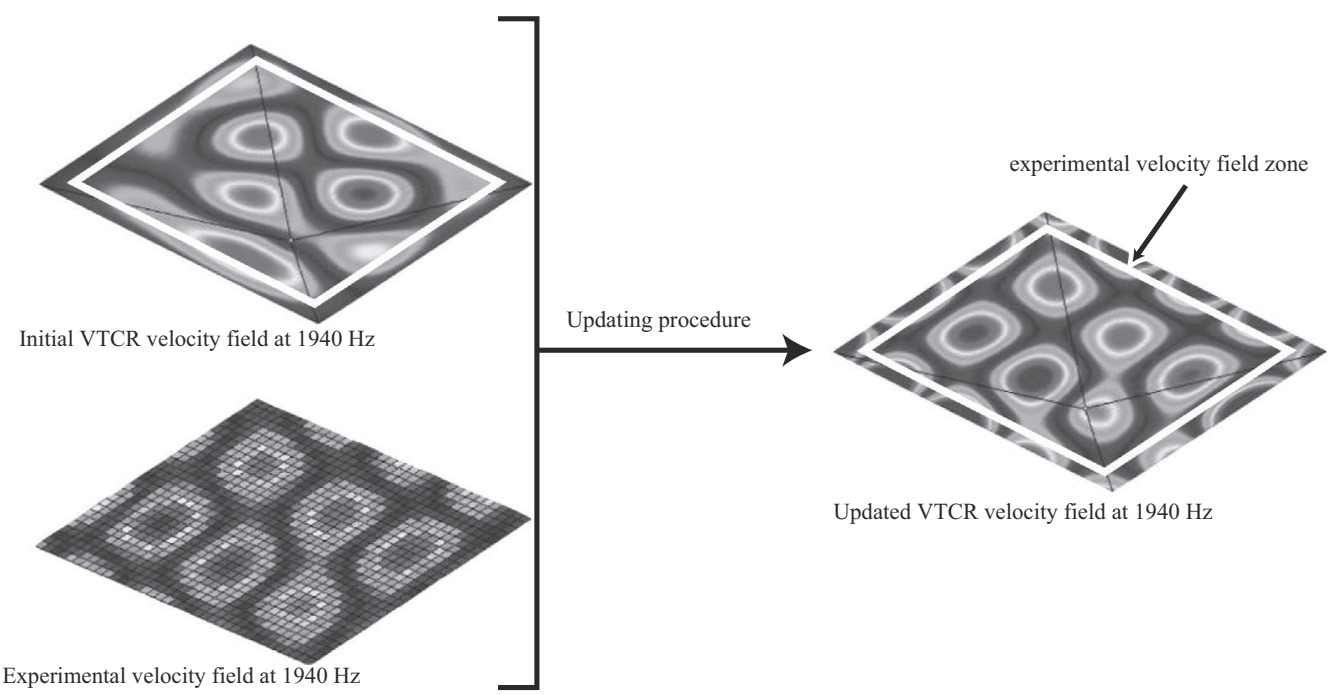

Fig. 14. Joints updating procedure at $1940 \mathrm{~Hz}$. 


\subsection{Identification of the nominal seals mechanical properties}

Identification tests will be carried out on a dedicated setup allowing various compression ratio including the nominal one (the setup will be used for the identification procedure and for the comparisons of probabilistic frequency response functions). A glass screen will then be clamped between two steel frames. The distance between the two frames will be precisely controlled by 24 cylindrical braces (represented in Fig. 12) held by the 24 screws. 6 lengths of braces $\left(l_{i}\right.$ for $i \in[1,6]$ ) permit to have access to 6 discrete compression ratio: $0,18,36,54,72$ and 90 percent.

A 4808 Brüel \& Kjær shaker connected to the screen with a 8001 Brüel \& Kjær impedance head will provide a white noise from 0 to $6.4 \mathrm{kHz}$ and an Ometron VPI 8330 laser vibrometer will scan the screen in order to measure the velocity field on the glass front face (the distance between the screen and the vibrometer is $2 \mathrm{~m}$ ). Force and acceleration signals coming from the impedance head will be conditioned using two 2635 Brüel \& Kjær charge amplifiers. The screen will be configured in a horizontal position in order to minimize the gravity effect. The measurement mesh used by the scanning vibrometer will contain $29 \times 38$ points (the spatial resolution is $5 \mathrm{~mm}$ ). Reciprocity tests with different shaker locations have shown that the screen has a linear behavior for a fixed compression ratio. Fig. 13 represents the experimental setup, a velocity field at $3500 \mathrm{~Hz}$ and a typical mobility function at a given point $M$.

Table 1

Identified seals properties.

\begin{tabular}{|c|c|c|c|c|c|c|c|c|c|c|}
\hline Frequency (Hz) & 106 & 356 & 560 & 863 & 1068 & 1940 & 2992 & 5068 & 7045 & 8333 \\
\hline$k_{t}\left(10^{6} \mathrm{~N} \mathrm{~m}^{-1}\right)$ & 0.13 & 0.43 & 0.67 & 1 & 1.2 & 2 & 2.8 & 4 & 4.7 & 5.1 \\
\hline$\eta_{\mathrm{t}}(\%)$ & 3 & 10 & 15 & 21 & 25 & 37 & 47 & 55 & 58 & 59 \\
\hline$k_{t}\left(10^{6} \mathrm{~N} \mathrm{~m} \mathrm{rad}^{-1}\right)$ & 0.1 & 0.55 & 0.73 & 1.06 & 1.38 & 3.44 & 2.84 & 7.07 & 5.86 & 9.71 \\
\hline$\eta_{\mathrm{t}}(\%)$ & 5 & 15 & 29 & 41 & 28 & 74 & 81 & 58 & 95 & 91 \\
\hline
\end{tabular}
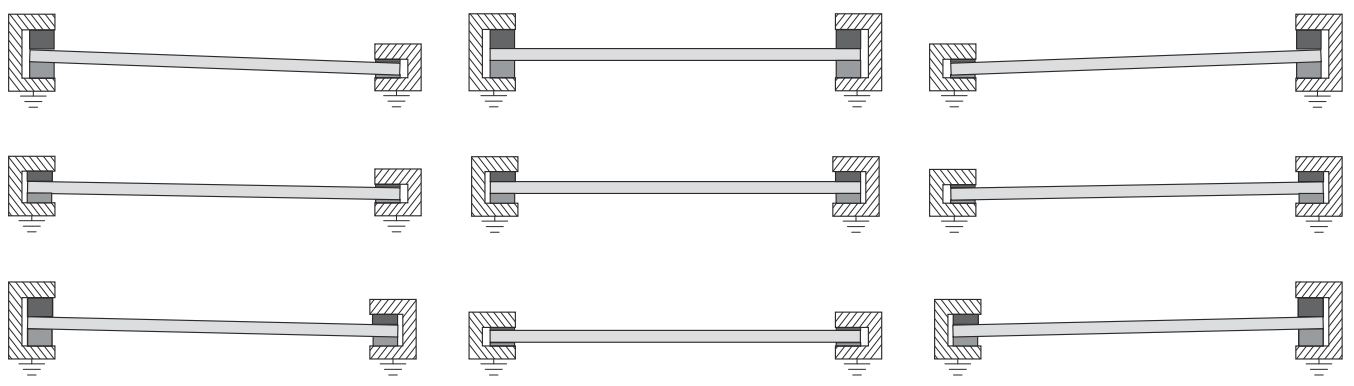

Fig. 15. Some of the possible setup configurations.

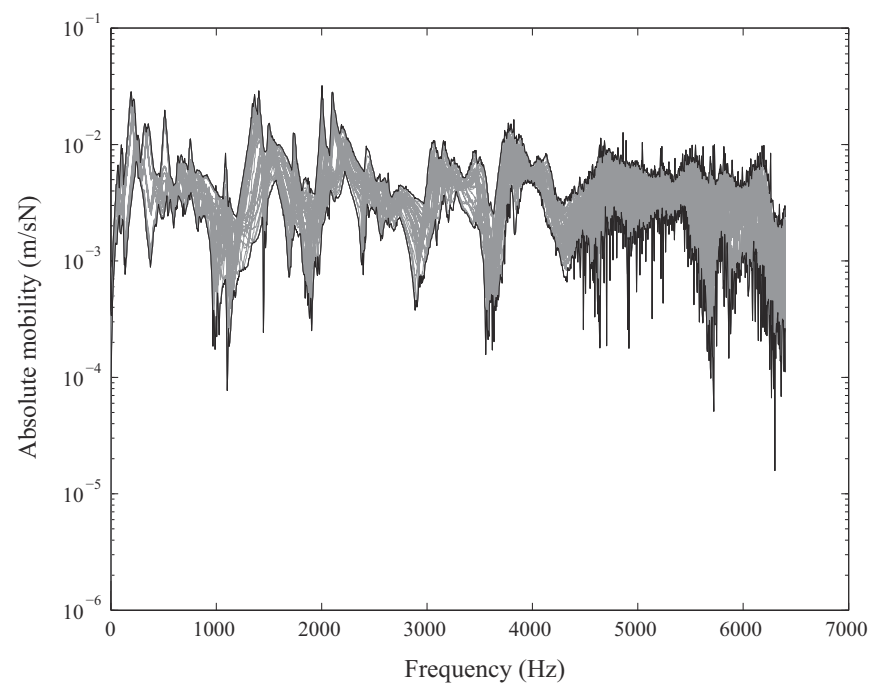

Fig. 16. Experimental variability domain at point $A$ for the 66 configurations. 
We will not detail the updating procedure of the seals as it has been already presented in the previous papers [28]. We just will recall that we use as initial value of the parameters those provided by the manufacturer of the seals and that a substructured version of the VTCR [29] is used in order to build a cost function composed of two terms: an interface residual (which quantifies the satisfaction of the interface equations) and a measurement term (which represents a distance between the experimental and numerical solutions). The updating procedure is an iterative process that will stop when the absolute error between the experimental velocity and the simulated velocity will be lower than 10 percent on each point of the experimental mesh. This updating procedure (Fig. 14) will be carried out for ten values of frequency (106, 356, 560, 863, 1068, 1940, 2992, 5068, 7045 and $8333 \mathrm{~Hz}$ ) associated to specific shape modes in order to have the evolution of the seal stiffness and damping as functions of frequency. The values of the seals parameters between these specific frequencies have been linearly interpolated.

Table 1 provides the values of the seals mechanical properties obtained from the updating procedure.

Once these nominal values are identified, we will associate with each parameter a random variable representing in a global way the fluctuations which could be related to various phenomena: (compression ratio, temperature, humidity, etc.). In this specific case, the randomness of the response comes only from the random behavior of the connections [10]: more precisely, the translational and rotational stiffnesses $\left(k_{t}\right.$ and $\left.k_{r}\right)$ and the translational and rotational loss factors $\left(\eta_{t}\right.$ and $\left.\eta_{r}\right)$ which may be uncertain, are modeled by a random variable.

\subsection{Experimental variability}

The setup presented in chapter 5.2 has also been used to provide probabilistic frequency response functions. By combining different length of the braces $l_{i}$ from the 6 possible one, it is possible to generate 66 setup configurations associated to various discrete compression ratio as various angulations. Some of them are presented in Fig. 15.

Even if 66 different configurations are possible, this setup is not able to generate variability on the four parameters individually. On an experimental point of view, the four parameters could be not as independent as we would like but we have assumed that with different size of brace, we will have a good representation of the variability domain.
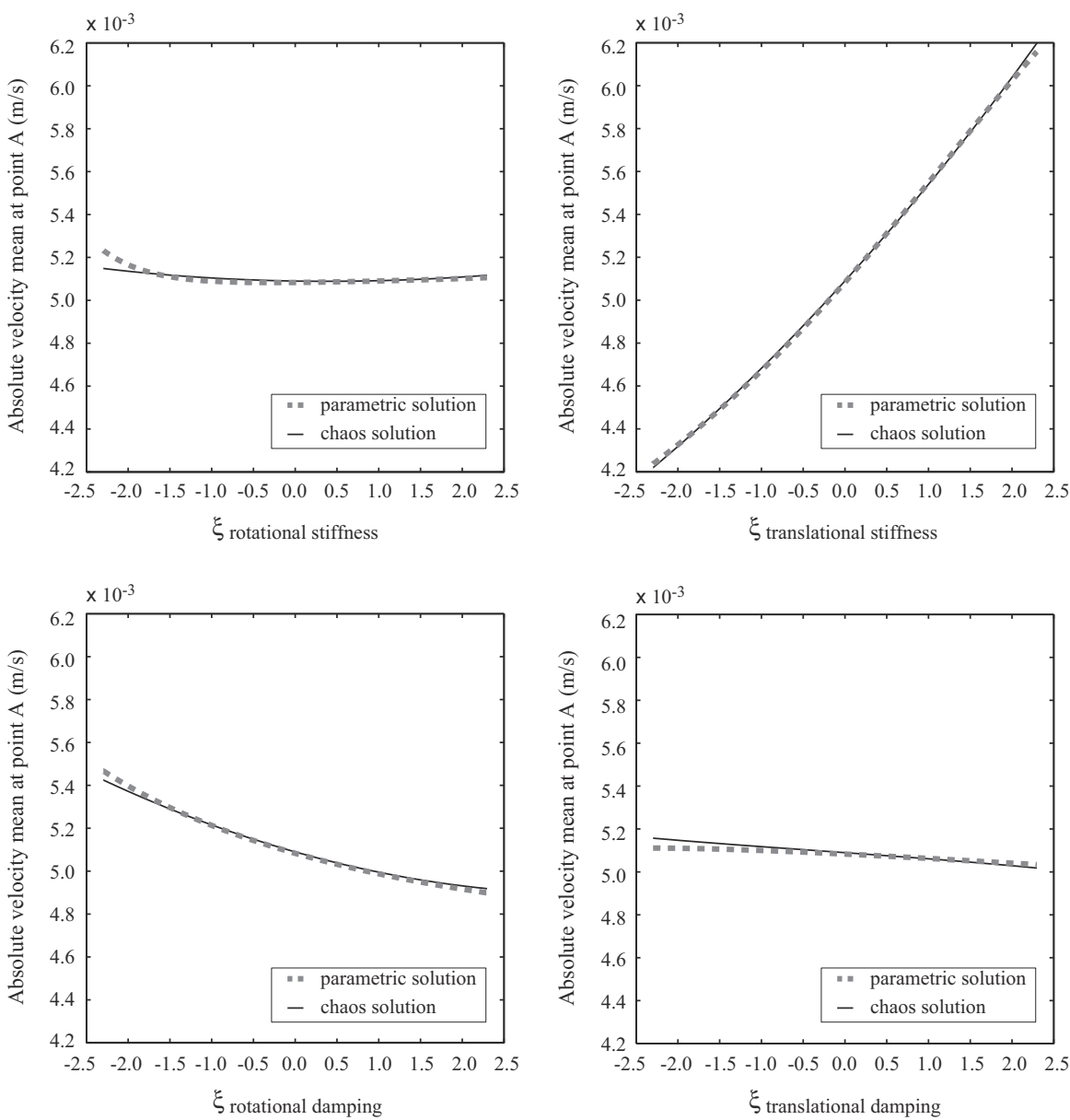

Fig. 17. Absolute velocity at point $A$ function of $\xi_{\mathrm{i}}$, the others $\xi_{\mathrm{j}}$ are equal to 0 . Comparison between the parametric and chaos solutions at $1940 \mathrm{~Hz}$. 
In order to build the experimental reference values, we have extracted the velocity in the vicinity of a dedicated point $A$. Its location related to the coordinate system defined in Fig. 10 is given by (200 mm, $30 \mathrm{~mm}$ ).

The measures have been done 2 times for each configuration. Fig. 16 gives the frequency response functions of these 132 measures (in gray) as the maximum and the minimum of them (in black) that will constitute our experimental variability domain.

\subsection{Numerical variability}

To compute the numerical variability, we have used for the seals mechanical properties mean values the ones provided in Table 1. The polynomial decomposition was conducted using second-order polynomial functions ( $p=2)$ with four independent stochastic parameters $(n=4)$. This simple model could be improved with experimental data and the potential stochastic dependencies between variables taken into account. The second-order decomposition leads to the computation of 15 values $\mathbf{a}_{j}$ associated with 15 Hermite's functions $\Psi_{j}(\theta)(j=1,15)$.

In order to compare the parametric resolution to the VTCR method with polynomial chaos, we show in Fig. 17 the velocity at the given point $A$ as a function of $\xi$ for a second order of polynomial chaos. As expected, the PCE yields a good approximation of the exact solution. The accuracy increases with the order of the PCE but the second order is sufficient thanks to the smoothness of the solution.

The VTCR-PCE solution has been calculated by subdividing the frequency domain [400, $6400 \mathrm{~Hz}]$ in 50 frequency steps. The number of draws needed for achieving convergence for each frequency step will be $10^{4}$ according to the curves presented in Fig. 18.

(a)

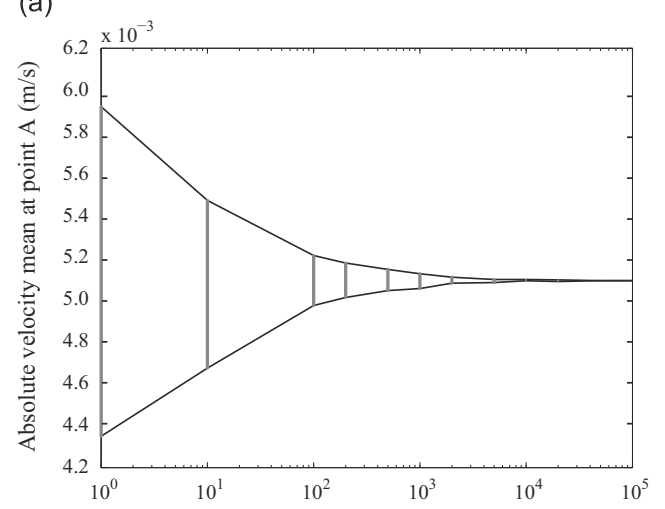

(b)

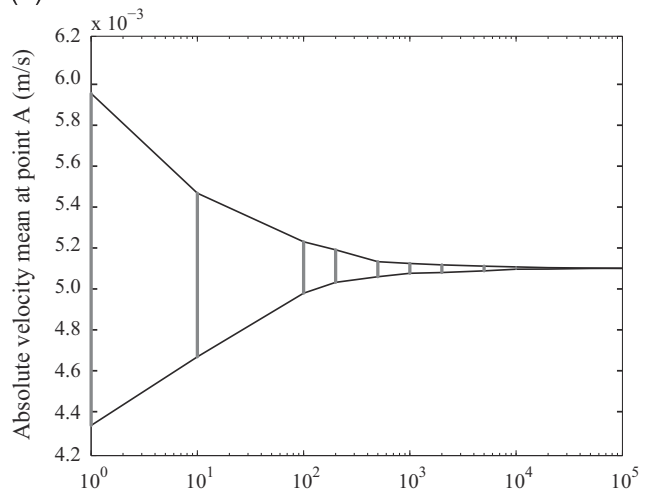

Fig. 18. Convergence of the absolute velocity and number of draws for exact calculation (a) and chaos representation (b) at $1940 \mathrm{~Hz}$.

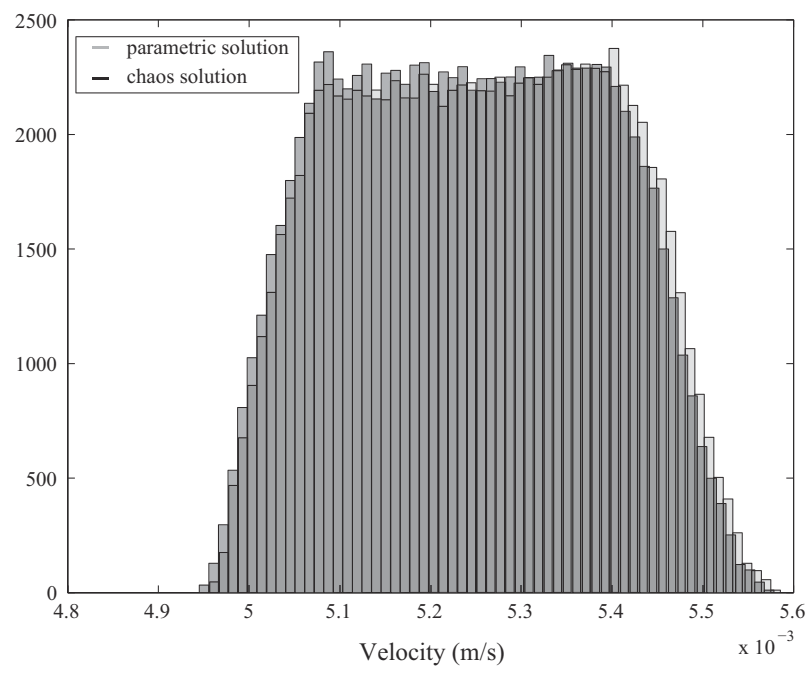

Fig. 19. Probability density functions of the velocity at point $A\left(\delta_{i}=0.25, i=1.4\right)$ : comparison between the Chaos solution and the Monte Carlo solution $(100,000$ draws $)$ at $1940 \mathrm{~Hz}$. 


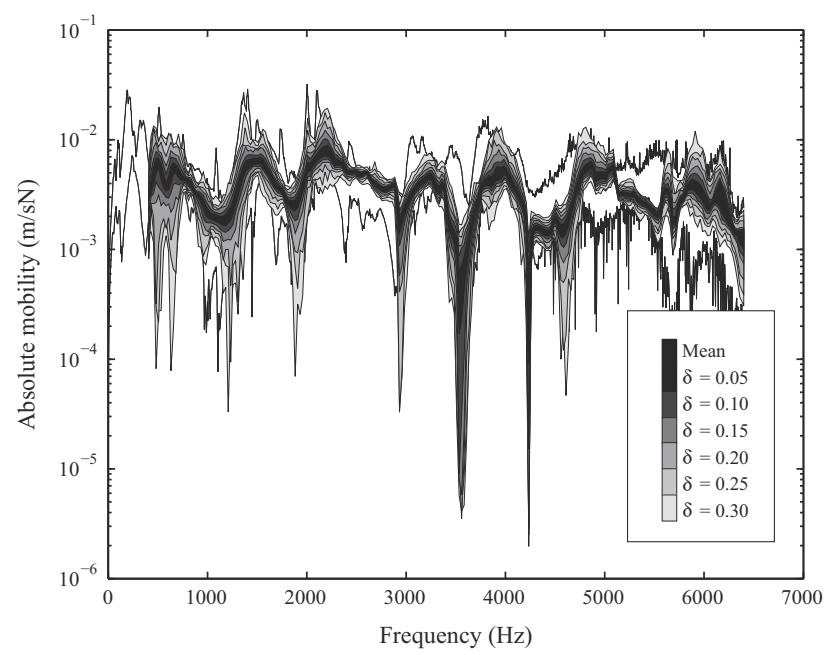

Fig. 20. Simulated variability for different values of coefficients of variation.

Fig. 19 shows that the comparison of the probability density function of the velocity at the point $A$ obtained by the VTCRChaos method with that obtained by a classical Monte Carlo method ( $10^{4}$ draws). The result obtained with the chaos representation is almost the same as those obtained by direct resolution.

Simulations will be computed for coefficients of variation going from 0.05 to 0.30 for the four parameters. With these values, we are able to generate a fluctuation on the parameters going from 50 to 200 percent. These simulated fluctuations are comparable to the ones observed in experiments on both stiffnesses (Fig. 11) but are smaller than the real ones for both damping parameters (identification procedures for high compression ratio have shown that the variation of these parameters is more a factor of ten).

Fig. 20 shows the simulated response of the structure including the stochastic interfaces. These simulations are in good agreement with the experimental variability domain. The main differences come from frequency bandwidths where the real variation of the damping generated by the compression ratio is much more important than the one generated by our associated random variables.

As the efficiency of the approach has been demonstrated on the real touch screen subjected to high variation on the joint parameters, this method can now be applied to weaker variabilities as the ones observed under the real conditions of operation. We are now able to provide the simulated stochastic frequency response functions that give us the different frequency bandwidths where the touch screen behavior is less sensitive to the joint parameters. These frequency bandwidths must be used in order to provide an accurate and robust detection procedure.

\section{Conclusions}

The Variational Theory of Complex Rays is a general approach to medium-frequency calculations. Its main features are the use of shape functions with a sound mechanical meaning and a dedicated variational formulation allowing the use of independent approximations in the different substructures. For complex structures, the VTCR has a good ability to give predictive results at a very low numerical cost. It can also take into account complex connections among substructures, described by stochastic parameters, in order to represent the physical reality at the lowest possible numerical cost. In this case, the benefit of two-scale shape functions is twofold: first, the global system defined by the PCE is relatively small; second, the smooth variation of the unknowns (generalized amplitudes) when the connection parameters vary can be easily represented by a polynomial function. This method has been tested successfully on a new kind of touch screen in order to simulate the influence of the seals mechanical properties on the vibrational signatures.

\section{References}

[1] W. Desmet, B. van Hal, P. Sas, D. Vandepitte, A computationally efficient prediction technique for the steady-state dynamic analysis of coupled vibro-acoustic systems, Advances in Engineering Software 33 (2002) 527-540.

[2] A. Deraemaeker, I. Babuska, P. Bouillard, Dispersion and pollution of the FEM solution for the Helmholtz equation in one, two and three dimensions, International Journal for Numerical Methods in Engineering 46 (1999) 471-499.

[3] R. Lyon, G. Maidanick, Power flow between linearly coupled oscillators, Journal of the Acoustical Society of America 34 (5) (1967) $623-639$.

[4] M. Chevreuil, P. Ladevèze, P. Rouch, Transient analysis including the low and the medium-frequency ranges of engineering structures, Computers and Structures 85 (17-18) (2007) 1431-1444.

[5] R. Ghanem, P. Spanos, Stochastic Finite Elements: A Spectral Approach, Springer, Berlin, 1991.

[6] A. Sarkar, R. Ghanem, Mid-frequency structural dynamics with parameter uncertainty, Computer Methods in Applied Mechanics and Engineering 191 (2002) 5499-5513. 
[7] R. Ghanem, S. Masri, M. Pellissetti, R. Wolfe, Identification and prediction of stochastic dynamical systems in a polynomial chaos basis, Computer Methods in Applied Mechanics and Engineering 194 (2005) 1641-1654.

[8] K. Sepahvand, S. Marburg, H.J. Hardtke, Stochastic free vibration of orthotropic plates using generalized polynomial chaos expansion, Journal of Sound and Vibration 331 (1) (2012) 167-179.

[9] J. Didier, B. Faverjon, J. Sinou, Analysing the dynamic response of a rotor system under uncertain parameters by polynomial chaos expansion, Journal of Vibration and Control 18 (2012) 712-732.

[10] C. Blanzé, P. Rouch, Analysis of structures with stochastic interfaces in the medium-frequency range, Journal of Computational Acoustics 13 (4) (2005) $711-729$.

[11] J. Wijker, Spacecraft Structures, Springer, 2008.

[12] A. Caignot, P. Ladevèze, D. Néron, J.F. Durand, Virtual testing for the prediction of damping in joints, Engineering Computations 27 (5) (2010) 621-644.

[13] M. Kleiber, T. Hien, The Stochastic Finite Element Method. Basic Perturbation Technique and Computer Implementation, John Wiley \& Sons, 1992.

[14] G.D.F. Yamazaki, M. Shinozuka, Neumann expansion for stochastic finite element analysis, Journal of Engineering Mechanics 114 (1988) $1335-1354$.

[15] N. Wiener, The homogeneous chaos, American Journal of Mathematics 60 (4) (1938) 897-936.

[16] R. Cameron, W. Martin, The orthogonal development of nonlinear functionals in series of Fourier-Hermite functionals, The Annals of Mathematics 48 (16) (1947) 385-392.

[17] R. Ghanem, R. Kruger, Numerical solution of spectral stochastic finite element systems, Computer Methods in Applied Mechanics and Engineering 129 (1996) 289-303.

[18] R. Ghanem, Ingredients for a general purpose stochastic finite elements implementation, Computer Methods in Applied Mechanics and Engineering 168 (1999) 19-34.

[19] D. Xiu, G.E. Karniadakis, Modeling uncertainty in steady state diffusion problems via generalized polynomial chaos, Computer Methods in Applied Mechanics and Engineering 191 (43) (2002) 4927-4948.

[20] E. Jaynes, Information theory and statistical mechanics, Physical Review 106 (4) (1957) 620-630.

[21] Y. Saad, M. Schultz, Gmres: a generalized minimal residual algorithm for solving nonsymmetric linear systems, SIAM Journal on Scientific and Statistical Computing 7 (3) (1986) 856-869.

[22] M. Pellissetti, R. Ghanem, Iterative solution of systems of linear equations arising in the context of the stochastic FEM, Journal of Advances in Engineering Software 31 (2000) 607-616.

[23] R. Bernhard, F. Milner, Vibrations of a beam and related statistical properties, Mathematical and Computer Modelling 34 (2001) $657-675$.

[24] G. Rabbiolo, R. Bernhard, F. Milner, Definition of a high-frequency threshold for plates and acoustical spaces, Journal of Sound and Vibration 277 (2004) 647-667.

[25] B. Bloss, M. Rao, Measurement of damping in structures by the power input method, Experimental Techniques 26 (3) (2002) $30-33$.

[26] E. Balmès, M. Corus, S. Germès, Model validation for heavily damped structures. Application to a windshield joint, ISMA06, International Conference on Noise and Vibration Engineering, Leuven, Belgium, 2006.

[27] J. Park, L. Mongeau, T. Siegmund, Influence of support properties on the sound radiated from the vibrations of rectangular plates, Journal of Sound and Vibration 264 (2003) 775-794.

[28] O. Dorival, P. Rouch, O. Allix, A substructured Trefftz method for updating joint models in the medium-frequency range, Computational Mechanics 42 (3) (2008) 381-394.

[29] O. Dorival, P. Rouch, O. Allix, A substructured version of the variational theory of complex rays dedicated to the calculation of assemblies with dissipative joints in the medium-frequency range, Engineering Computations 27 (7) (2006) 729-748. 